\title{
Bacteriological complications from the use of urethral instruments: Principles of prevention ${ }^{1}$
}

\author{
J. P. MITCHELL AND W. A. GILLESPIE \\ From the Departments of Urology and Bacteriology, \\ United Bristol Hospitals and University of Bristol
}

SYNOPSIS Guidance by bacteriologists and changes in theatre and ward disciplines have greatly. improved the results of urological surgery in recent years. The most noteworthy successes have been: in treatment of chronic retention with renal failure, and in prevention of the bacteraemia which may occur following removal of the post-operative catheter or the passage of an instrument to은 dilate a stricture. It is hoped that safer urethral instrumentation may also reduce the incidence of $\bar{\oplus}$ chronic pyelonephritis.

\section{PART I: THE PROBLEMS AS SEEN BY THE SURGEON}

Many papers have been written about urinary tract infection, but the risks of contamination by instruments passed along the urethra have received little attention until recently. The immediate acute symptoms of instrumentally-induced infection in the male are easily recognized, for example, acute prostatitis and epididymitis. Though many infections clear up without causing great harm, the consequences may be serious as the following brief history illustrates.

A patient had a transurethral resection of the prostate and bladder neck performed some years ago. Following the operation, the urine was infected with Proteus, which was not eradicated. However, because pus had disappeared from his urine, it was assumed that his natural resistance had overcome the infection and there was no need to follow him any longer. Within nine months of his original operation, bilateral renal calculi had developed (Fig. 1). Proteus is a potential stone former, with a predilection for the urinary tract, but fortunately it is unusual for a stone to be produced so quickly.

Until recent years, some infection following prostatectomy was regarded as inevitable. In fact, some of the older surgeons practising urology used to say that a 'touch of infection' of the urinary tract was an advantage. This impression probably arose from the results of treating patients with chronic retention of urine. A large volume of uninfected urine remaining in the bladder after micturition

${ }^{1}$ Based on two papers read at the April meeting of the Association of Clinical Pathologists. always carries a serious risk, because of the possible $\frac{\mathbb{\Phi}}{\sim}$ associated hydronephrosis and renal failure. The $\vec{\theta}$ kidneys had so far withstood the back pressure, but $\stackrel{\AA}{\perp}$ not infection. Surgery, or even the simplest procedure, such as the passage of a catheter, was sufficient to introduce infection, adding a further burden to the already damaged kidneys, with the result that theo

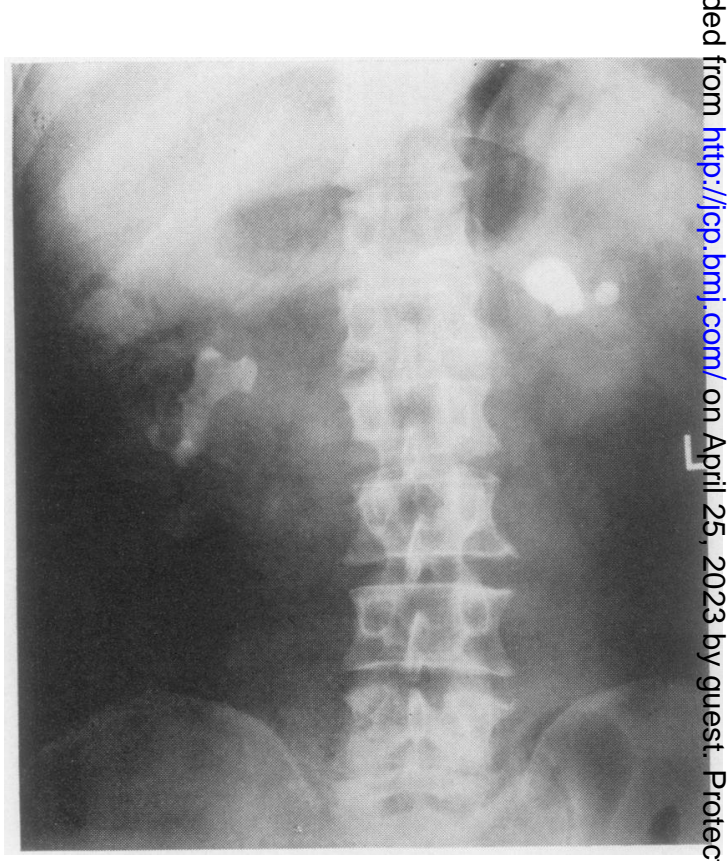

FIG. 1. Bilateral renal calculi after Proteus infection ha persisted for only nine months. 
patients went downhill and many died from ascending urinary infection and acute pyelonephritis. The patient who came for treatment of chronic retention, having already survived an infection, was in a stronger position. Drainage of the bladder produced nothing but benefit because the kidneys had already withstood both back pressure and urinary infection. The catheter relieved the pressure and gave such a patient the chance to combat the infection. It was for this reason that the older surgeons gained the impression that 'a touch of infection' was an advantage. This stage has been passed, as nowadays we can often avoid infection altogether. Our aim now is to drain the uninfected chronic retention, taking meticulous care to preserve a sterile urine until the kidneys recover.

Another example of infection of the urinary tract, which in the past has been accepted as an inevitable hazard, was in regular attenders at urethral stricture clinics. These men were accustomed to having a rigor during the evening following dilatation of the stricture. By the next morning they usually felt fit to return to work. Scott (1929) and Barrington and Wright (1930) showed that the attacks were due to bacteraemia. Mitchell, Slade, and Linton (1962) confirmed that these patients were suffering from bacteraemia and described methods of introducing disinfectants into the urethra and bladder (see part II); their investigations were stimulated by a tragic episode which befell one patient with a traumatic stricture who, as an out-patient, had been treated periodically by dilatation. On returning home after a difficult dilatation he had a rigor far worse than any previous one, but unfortunately did not call his doctor for 48 hours. By this time a diagnosis of acute coronary occlusion was made, and the patient was admitted to a medical ward. He proved, in fact, to have a fulminating septicaemia caused by a coliform bacillus. He lost one eye from panophthalmitis, had several metastatic abscesses, and a spondylitis which took 18 months to settle. A tragedy such as this must be very rare, but it emphasizes the need to investigate the rigors and if possible prevent them.

Another example of a similar nature is the socalled 'catheter fever', when a patient, following an uneventful prostatectomy, develops slight pyrexia within a few hours of the removal of the catheter. In the days when most prostatectomies were followed by infection, this was a remarkably common occurrence. It was shown that blood culture taken immediately after removal of the catheter was positive in almost half the patients with infected urine but not in patients with sterile urine (Slade, 1958). The urethral mucosa had become hyperaemic due to the presence of the catheter, and after the catheter was removed, the inflamed mucosa allowed organisms to pass into the blood stream. This bacteraemia often seemed to occur with the first act of micturition, and was found to be aggravated if the patient had the additional pelvic disturbance of a bowel action at the same time.

Catheter-induced bacteraemia, though often transient and symptomless, not infrequently causes brief pyrexia and rigor. Occasionally the consequence is much more serious and has been aptly described as bacteraemic shock (Waisbren, 1951). The responsible organism is usually a Gram-negative bacillus. The clinical picture is usually a febrile toxic condition, but it can also present with little or no fever in a very ill patient whose sudden fall of blood pressure suggests a cardiovascular accident. In fact, it is these cases of hypotensive febrile bacteraemia which require the most urgent and effective treatment and the improvement can then be equally rapid and dramatic. The sudden collapse of a urological patient should warn the clinician that the cause may be bacteraemia. The most important step in treatment is to inject one or more antibiotics which will be likely to deal with prevalent cross-infecting organisms (British Medical Journal, 1964).

The danger of catheter-induced infection in females has not been appreciated until recently. In the male, catheterization was known to carry some danger, because of the epididymitis and prostatitis which sometimes followed it. In the female, on the other hand, acute immediate symptoms rarely followed the passage of a catheter and therefore its dangers escaped notice. Not only were women catheterized to obtain urine for the laboratory, but also it was the practice in labour wards to pass a catheter just before delivery to make sure that the bladder was empty. Gynaecologists have used catheters repeatedly; in fact, catheterization was almost an essential part of a pelvic repair. Many gynaecological patients were either catheterized repeatedly after operation or had an indwelling catheter for several days. Specimens of urine from such patients with open-draining indwelling catheters showed that $97 \%$ became infected (Gillespie, Lennon, Linton, and Slade, 1964).

We know that in women a urinary infection sometimes appears for the first time spontaneously. Furthermore, if the urinary infection produces any symptoms and the patient is then treated with antibiotics, it usually clears up. But some infections in women do not respond to treatment or recur soon after the antibiotic is withdrawn. Presumably there is a risk that infection in the lower urinary tract, whatever its origin, may ascend to the kidney. How often, then, may pyelonephritis have resulted from indiscriminate catheretization of a woman either to 
collect a specimen of urine, or as a preliminary to childbirth?

When it was realized that the infections which so commonly followed operation and catheterization were sometimes harmful, methods of avoiding them were introduced. These were the avoidance of unnecessary catheterization, efficient sterilization of instruments, disinfection of the urethra at the time of operation or catheterization, and maintenance of sterility during the period of catheter drainage. These methods are dealt with in part II but it is appropriate at this point to emphasize the peculiar difficulties of sterilizing cytoscopes. Everyone would agree that disinfection by heat is most reliable but although the cement of lens mountings can bemade to withstand temperatures of $80^{\circ} \mathrm{C}$. or $100^{\circ} \mathrm{C}$., there are still certain telescopes such as those used in panendoscopes and in children's cystoscopes in which the more heat-resistant cements cannot be used. The fine insulation in the cystoscope sheath carrying the cable to the cystoscope lamp is also liable to perish with repeated heat changes. Finally, the air trapped inside the telescope will expand and contract with temperature changes and put an additional strain on the lens mountings. Autoclaving a cystoscope is therefore unsafe, and it seems doubtful whether any perfect method of sterilizing a cystoscope has yet been devised, though the best is some form of pasteurization as described by Francis (1959).

The speed of sterilization is also important. So long as only the standard cystoscope is required, no delay should be experienced, because these instruments can be duplicated in the theatre in sufficient numbers to allow a reasonable time for sterilization. On the other hand, a urologist starting an endoscopic list does not know for certain what instruments he will require beforehand. To sterilize every endoscopic instrument before starting on a cystoscopy list would be to subject some of the less commonly used instruments to unnecessary trauma. The best example is endoscopy in children, when a variety of sizes of instruments may be called for at short notice. For this reason, a rapid method of sterilizing cystoscopes is necessary.

The obvious answer to this problem is storage of all endoscopy instruments in a sterile state. In the past, this has been attempted by the formalin cabinet but this has obvious bacteriological shortcomings in addition to the conjunctival discomfort of the surgeon and the danger of irritating the patient's urethra. The formalin-sterilized cystoscope still smells of formalin however much it is rinsed or wiped. In a nutshell, then, what is required is a rapid effective method of sterilization which will not damage the instruments and some means of storing them indefinitely in a sterile state.
PART II: BACTERIOLOGICAL PRINCIPLES IN THE PREVENTION OF INFECTION

Prevention should be based on knowledge of how bacteria reach the bladder of the urological or $\overline{\bar{p}}$ gynaecological patient. The organisms may comed from the patient, by self-infection, or from another patient, by cross-infection (Fig. 2). The most ${ }^{\infty}$ important source of cross-infection is the urine of $\vec{O}$ another patient, from which organisms may be $\overrightarrow{\vec{H}}$ transferred by cystoscopes, urine bottles, etc. O However, with the exception of the patient witho suprapubic drainage, there is only one final portal? of entry which the organisms must pass, the urethra.. Bacteria may enter the bladder at the moment of inserting the cystoscope or catheter. They mayse come from the instrument, if it is not sterile, oro from the urethra, which is never sterile. If a catheter? is left indwelling, it may permit entry of bacteria incs two more ways. First, with open drainage, bacteriad multiplying in urine in the collecting vessel will contaminate the lower end of the catheter and thences be carried up to the bladder by reverse currents of $\frac{\mathbb{D}}{-}$ fluid propelled by rising air bubbles, and perhaps $\overrightarrow{0}$ in other ways. These organisms will often be crossinfecting strains. The second route is mainly one of self-infection, and is the space between the cathetero and urethral wall. The short female urethra permitsthe catheter to move when the patient moves. This 'extra-catheter' route is probably less important in men.

These facts suggest two approaches to the problem $\overrightarrow{\vec{B}}$ of preventing infection. One is to exclude bacteria from the urethra, and the other is to destroy them as soon as they enter the bladder. For these purposes: local disinfectants are preferable to systemic chemo therapy with its tendency to breed resistant strains In Bristol we rely on asepsis and closed bladder. drainage, supplemented by local antisepsis.

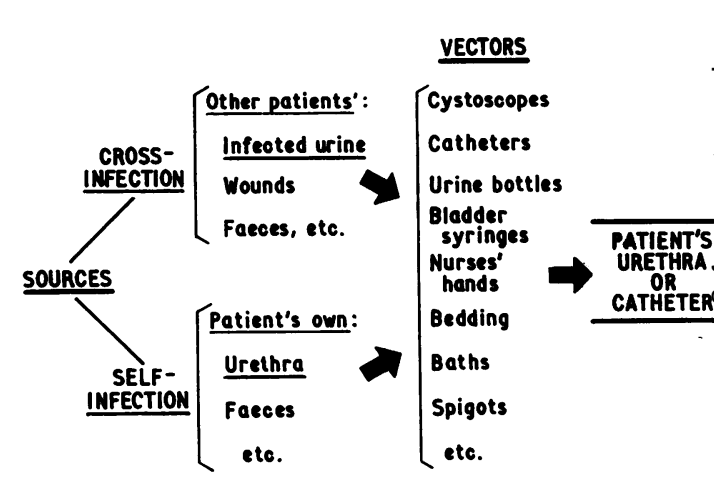

FIG. 2. Sources and routes of urinary infection. 
METHODS OF PREVENTION IN WOMEN With intermittent catheterization the risk of infection may be reduced by disinfecting the urethra before passing the catheter. Figure 3 shows two groups of patients who were repeatedly catheterized after gynaecological operations. In the control group, the urethra was lubricated with a non-disinfectant jelly, and in the test group, with Hibitane (chlorhexidine) obstetric cream three minutes before each catheter insertion. The incidence of urinary infection was moderately reduced. The reduction was entirely in Gram-negative bacilli. Infections by Streptococcus faecalis, which is less sensitive than coliform bacilli to chlorhexidine, were unchanged (Gillespie, Lennon, Linton, and Slade, 1962).

Chlorhexidine may also be used by instilling a weak solution into the bladder through the catheter, as described by Paterson, Barr, and Macdonald (1960). We now use both methods.

Indwelling catheters nearly always cause infection if open drainage is used. In a series of gynaecological patients who were treated with open-drainage indwelling catheters for four days after operation, and subsequently were catheterized intermittently to measure residual urine, $97 \%$ developed urinary infection. Infection usually began during the period of indwelling catheter drainage. The causative pathogens ascended to the bladder both inside and outside the catheter. The few patients who survived this stage without infection became infected when intermittently catheterized afterwards. However, since an indwelling catheter is much pleasanter for patients and nurses than frequent intermittent catheterization throughout the post-operative period,

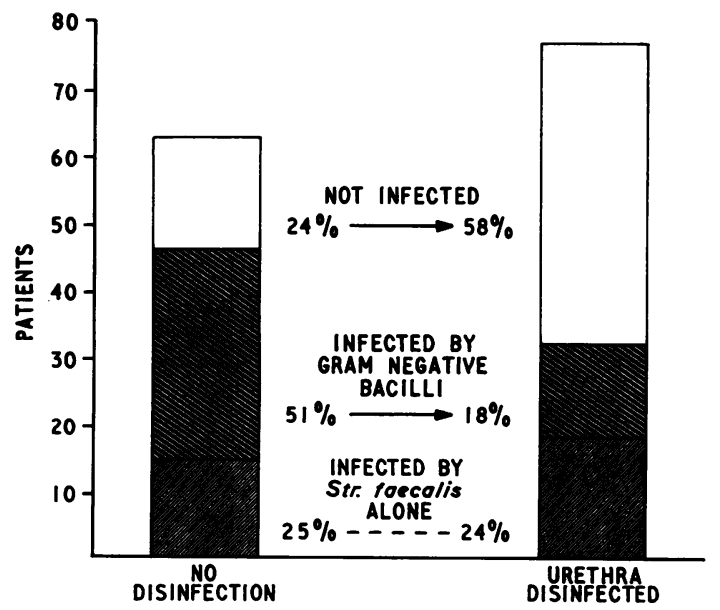

FIG. 3. Prevention of urinary infection by urethral treatment with chlorhexidine. three modifications were instituted in order to make it safer. (1) The risk of infection at the moment of inserting a catheter was reduced by disinfecting the urethra before insertion and the bladder immediately afterwards, by the methods already described. (2) Closed drainage into vessels containing disinfectant was employed. (3) The indwelling Foley catheter was modified by a device which prevented entry of organisms between the catheter and urethral wall. This was a collar of polyurethane plastic foam, tightly threaded on to the catheter and pushed up against the external urethral meatus as soon as the catheter was inserted and its retaining bulb inflated. The sponge collar was smeared twice daily with chlorhexidine cream so as to maintain a disinfectant barrier at the urethral meatus. Each of these modifications brought some improvement, and when they were used together for two years, the postoperative urinary infection rate averaged $13 \%$ compared with the previous rate of $97 \%$ (Gillespie et al., 1964).

Hannah (1963) obtained a similar improvement by using different methods. It is therefore clear that post-operative infection is generally preventable, even when indwelling catheters are used.

The change from open to closed drainage was followed by an interesting change in the predominant infecting bacteria. Infections during open drainage were caused by a variety of organisms, including Proteus and Pseudomonas, but the commonest were Klebsiellae, many of which were antibiotic-resistant. This finding was in line with results of other workers, some of whom have suggested that Klebsiellae develop drug resistance more easily than Escherichiae (Dutton and Ralston, 1957; Talbot, Cunliffe, and Gower, 1957; Lattimer, Seneca, Zinsser, and Troc, 1959). It was therefore interesting to find that when closed drainage was substituted for open drainage, thus reducing cross-infection, though still allowing self-infection by the patients' own flora, Klebsiellae became rare, and Escherichiae common (Gillespie et al., 1964). Escherichiae also predominated in other circumstances where cross-infection was unlikely, for example, in infection acquired outside hospital and in infections following intermittent catheterization.

METHODS OF PREVENTION IN MEN The value of sterile drainage into a closed vessel containing disinfectant was demonstrated some years ago in men with acute retention of urine whose bladders were drained for short periods (Miller, Linton, Gillespie, Slade, and Mitchell, 1960b). Recently we have investigated the use of carefully supervised closed drainage in 32 men whose bladders were drained for much longer periods (Fig. 4). Some 


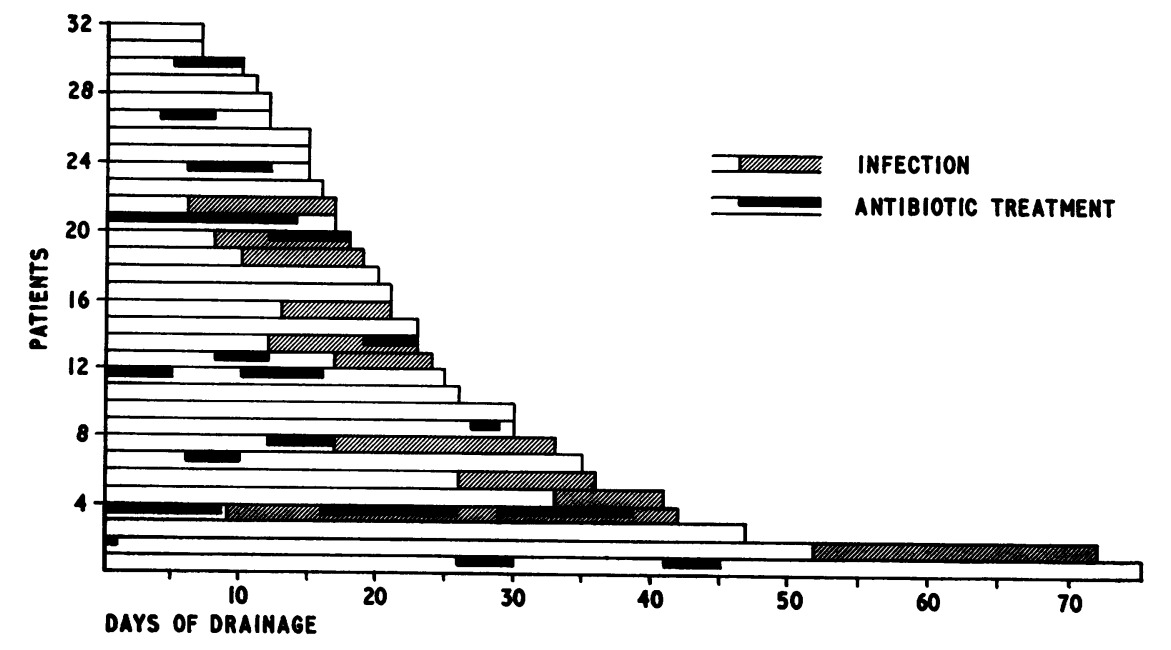

FIG. 4. Urinary infections in men treated with closed drainage.

patients were given antibiotics for respiratory infection, but this did not greatly influence the results. The drainage treatment was closely supervised to ensure that asepsis was maintained, especially when changing bottles. Many patients were kept free from infection for several weeks. When infection did occur, it was often attributable to elementary faults in technique. Previous experience showed that with open drainage all these patients would have been infected within a few days, but it was not considered justifiable to include a control group with this series.

More complicated methods were needed to prevent infection after prostatectomy by the transurethral and retropubic methods. In addition to closed drainage, the urethra was disinfected before operation, disinfection of cystoscopes was improved, and a device for irrigating the bladder without interrupting closed drainage was adopted (Miller, Gillespie, Linton, Slade, and Mitchell, 1958). The results of trials carried out in two hospitals between 1956 and 1959 showed a reduction of the infection rate from an average of $82 \%$ to $11 \%$ (Miller, Gillespie, Linton, Slade, and Mitchell, 1960a). The same methods have been in routine use since 1959, but are less closely supervised by the laboratory. Results are checked by quantitative cultures of two specimens of urine from each patient, the first obtained at operation and the second five to seven days later. Although some specimens have been missed, sufficient pairs have been cultured to allow the infection rates in one of the hospitals to be calculated each year since 1959 (Table). The results were not quite as good as during the period of close laboratory supervision but remained much better than before the new methods were adopted.
TABLE

URINARY INFECTION AFTER TRANSURETHRAL AND RETROPUBIC PROSTATECTOMY AT BRISTOL ROYAL INFIRMAR 迎 1955-1964

\begin{tabular}{llr} 
Date & Special Precautions (see text) & Infection Rato \\
\hline $1955-56$ & None & $42 / 57(74 \%)$ \\
$1958-59$ & Full precautions, closely supervised & $12 / 89(13 \%)$ \\
1960 & Full precautions, routine & $5 / 38(13 \%)$ \\
1961 & Full precautions, routine & $8 / 53(15 \%)$ \\
1962 & Full precautions, routine & $18 / 92(20 \%)$ \\
1963 & Full precautions, routine & $17 / 73(23 \%)$ \\
1964 & Full precautions, routine & $5 / 28(18 \%) \stackrel{0}{\mathbb{D}}$ \\
(4 months) & &
\end{tabular}

${ }^{1}$ Infections by Staph. albus were excluded (Mitchell, 1964).

Finally, we must consider how to prevent. bacteraemia which, in patients with infected urine often follows the dilatation of urethral strictures:The only certain method of prevention would be to avoid urinary infection. But when the urine is already infected can anything be done, in additions to, or instead of, prescribing systemic chemotherapyo with all its disadvantages? Recent studies have shown the value of disinfecting the urethra with chlorhexidine before dilating the stricture and disinfecting the bladder immediately afterwards before the patient passes urine. In a controlled triah blood cultures were taken from patients with infected urine five minutes after dilatation an $\alpha^{2}$ after subsequent micturition (Mitchell, Slade, and Linton, 1962). The unprotected patients experience $\mathscr{E}$ a very high incidence of bacteraemia, $39 \%$ aftere dilatation and $75 \%$ after subsequent micturition. The bacteraemia was transient and usually caused no symptoms. Urethral disinfection lowered the incidence्p of positive blood cultures after dilatation from $39 \%$ to $10 \%$; disinfection of the urethra or bladder, of 
both, reduced the post-micturition bacteraemias from $75 \%$ to $10 \%$. These findings are further evidence of the value of using local antisepsis to supplement aseptic techniques. The methods are essentially simple, but require attention to details by nurses who understand the underlying principles. Otherwise, the methods will fail. Standards can best be maintained by regular courses of instruction for nurses.

\section{REFERENCES}

Barrington, F. J. F., and Wright, H. D. (1930). J. Path. Bact., 33, 871. British medical Journal (1964). Editorial, 1, 254.

Dutton, A. A. C., and Ralston, M. (1957). Lancet, 1, 115.

Francis, A. E. (1959). Proc. roy. soc. Med., 52, 998.
Gillespie, W. A., Lennon, G. G., Linton, K. B., and Slade, N. (1962). Brit. med. J., 2, 13.

Hann , J ,, (1964). Ibid., in the press.

Lattimer, J. K., Seneca, H., Zinsser, H. H., and Troc, O. (1959). J. Amer. med. Ass., 170, 938.

Miller, A., Gillespie, W. A., Linton, K. B., Slade, N., and Mitchell, J. P. (1958). Lancet, 2, 608.

$-,-,-,,-, \quad(1960$ a). Ibid., 2, 886.

, Linton, K. B., Gillespie, W. A., Slade, N., and Mitchell, J. P. (1960b). Ibid., 1, 310.

Mitchell, J. P., Slade, N., and Linton, K. B. (1962). Brit. J. Urol., 34, 454.

Mitchell, R. G. (1964). J. clin. Path., 17, 105.

Paterson, M. L., Barr, W., and Macdonald, S. (1960). J. Obstet. Gynec. Brit. Emp., 67, 394.

Scott, W. W. (1929). J. Urol., 21, 527.

Slade, N. (1958). Proc. roy. soc. Med., 51, 331.

Talbot, J. M., Cunliffe, A. C., and Gower, N. D. (1957). J. clin. Path., $10,222$.

Waisbren, B. A. (1951). Arch. intern. Med., 88, 467. 